\title{
Use of Trochar Intercostal Drain as Tunneller in Hydrocephalous: A Novel Technique
}

\author{
Authors \\ Chaurasia Basant MD ${ }^{1}$, Sharma Nitin, MS, M.Ch. ${ }^{2}$, Memon MA, MS, M.Ch ${ }^{3}$ \\ Sharma Mini, $\mathrm{MD}^{4}$ \\ ${ }^{1}$ Associate Professor, Anaesthesia, CCM Medical College, Durg, CG \\ ${ }^{2}$ Assistant Professor, Paediatric Surgery, Pt JNM Medical College Raipur, formally Assistant Professor \\ Pediatric Surgery at AIIMS, Raipur and CM Hospital and Associated Medical College, Bhilai CG, India \\ ${ }^{3}$ Professor and Head, Paediatric surgery, Pt JNM Medical College Raipur, CG, India \\ ${ }^{4}$ Assistant Professor, Pt. JNM Medical College, Raipur, CG, India \\ Dr Basant Chaurasia \\ Associate Professor, Department of Anaesthesia, CCM Medical College, Durg, CG, India \\ Email:drbasantc@gmail.com,Ph.: 9425191791
}

\section{Introduction}

Ventriculoperitoneal shunt insertion is a common procedure in the management of hydrocephalous. The creation of subcutaneous tunnel is required in the process of insertion of shunt. Stainless steel tunneller is used in creation of the subcutaneous space. This report highlights the use of trocar intercostal drain in the creation of subcutaneous space.

\section{Aims}

To assess the use of trocar intercostal drain as tunneller in insertion of the VP shunt.

\section{Material and Methods}

All the patients of hydrocephalous presenting to the paediatric surgery department of CM Hospital and associated medical college, Bhilai and AIIMS, Raipur who underwent the ventriculoperitoneal shunt surgery using this technique were included in this study. The study duration was from June
2014- Feb 2017. Those where the data was incomplete, those refusing consent for shunt surgery or those where the tunnel could not be created and shunt insertion was done using other alternative techniques were excluded. The diagnosis of hydrocephalous was done using the ultrasound cranium or plain CT head whichever was available. A semi-circular incision was given over the right parietal region. Subcutaneous space creation was started using mosquito forceps. Number 16/20 intercostal drain with trocar was inserted in the shallow space created (Figure 1) and advanced under guidance towards the right hypochondrium (Fig 2) just like the stainless steel tunneller is inserted. A stab incision was given over the drain, the abdominal end of the shunt was inserted through the drain after removal of the trocar to guide it downwards after cutting the conical sharp end of the drain, once the shunt end reached the lower end the intercostal drain was 
removed (figure-3) and the remaining procedure was completed as it is done usually.

Study Design: Retrospective

\section{Results}

A total of 21 cases presented to us with hydrocephalous during the study duration out of which 16 cases underwent the surgery using this technique and they formed the study group. The mean age at presentation was 2 month (range 1-9 month). Mean duration of surgery was $41 \mathrm{~min}$ (range30-60 min). Mean duration of hospital stay was 3 days (range2-5 days). There were 11 males and 5 females. All the cases operated were of hydrocephalous due to congenital aqueductal stenosis. 15 were primary cases while 1 was a redo case presenting with shunt block operated elsewhere. The incidence of shunt block was seen in none of our patients. The average follow up was 3 month (Range 2-9 month).

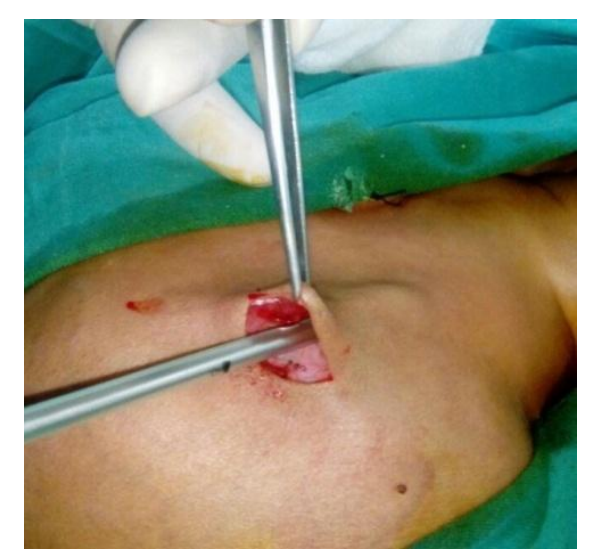

Figure 1 Insertion of trocar intercostal drain in the skull flap

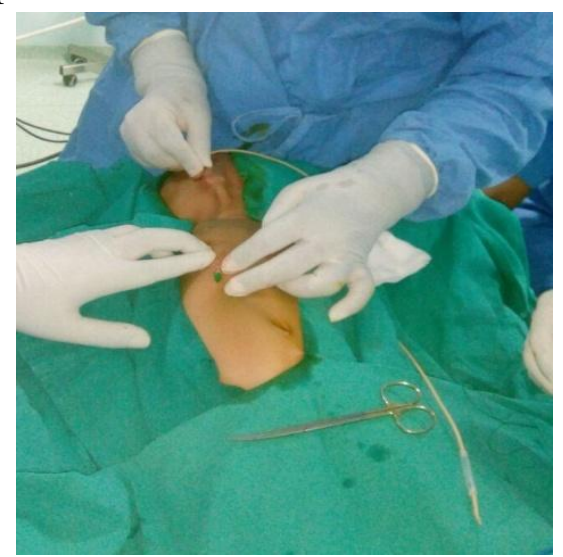

Figure 2 Completion of tunnel with intercostal drain coming out in hypochondrium

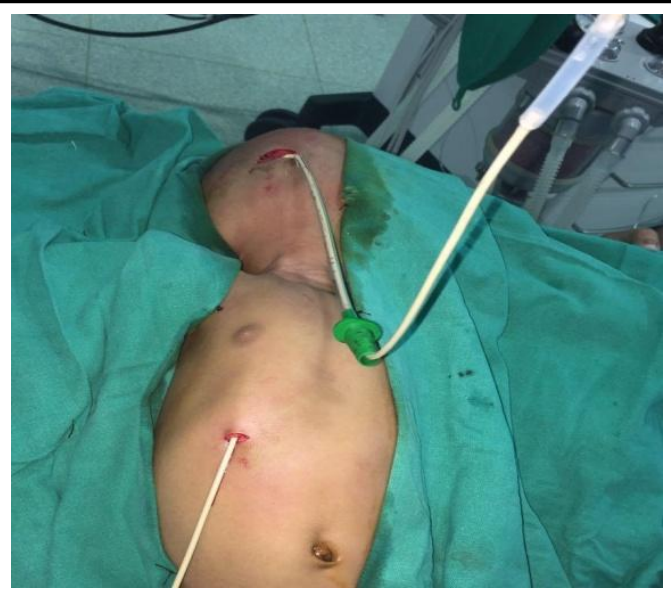

Figure 3 Removal of intercostal drain

\section{Discussion}

Hydrocephalus is associated with the accumulation of excessive CSF in the ventricle of the brain. This accumulation of the fluid/CSF occurs due to the imbalance between the formation and absorption of CSF in the brain. This imbalance leads to the accumulation of fluid which leads in an elevation in the intracranial pressure. Magendie and Lusckha described the communication between ventricular system and subarachnoid spaces. Key and Retzuis in 1872, Dandy and Blackfen in 1913 and 1914 and Weed in 1920, demonstrated that this can occur due to obstruction of the ventricular system. Hydrocephalous can thus be divided into two types:

a. Obstructive type due to blockage

b. Communicating type which is secondary to the decreased absorption over the surface of brain. ${ }^{(1)}$

There are various ways of managing hydrocephalous which ranges from medical management for low pressure system to surgical intervention in high pressure system. Surgical procedures available for the treatment of hydrocephalous include endoscopic third ventriculostomy and shunt surgery. Shunt surgeries are commonly performed for hydrocephalous because of the lack of availability of facilities for third ventriculostomy in all the centres. Most commonly performed shunt procedure for hydrocephalous is ventriculoperitoneal shunt. Some surgeons insert the shunt 
in any patient with large ventricles while others restrict it to those with potentially reversible deficit or progressive deterioration. ${ }^{(2)}$

There are three important steps in the insertion of ventriculoperitoneal shunt

1. Insertion of ventricular end.

2. Creation of subcutaneous tunnel for the placement of shunt tubing.

3. Insertion of abdominal end.

Ventricular end is inserted by creating a burr hole in the parietal bone. In our case the blunt end of the number 15 blade was used for the creation of the burr hole. (So that it could be reproducible in any of the setup even where the facility of burr is not available. The subcutaneous tunnel is created from skull to the anterior abdominal wall retroauricular. A number of techniques are used for the creation of subcutaneous tunnel, most commonly used instrument is a stainless steel tunneller which is reusable and available in various lengths. Two types of tunnellers are available one with a sheath and a trocar where the trocar is removed and shunt is inserted through the sheath; second tunneller is a rigid tunneller with a hole at the end and a silk thread is tied at its end and pulled up to aid in the guiding the lower end of shunt in the subcutaneous plane.

In situation where tunneller is not available a number of alternate ways are available vis. creation of subcutaneous space by small incisions in step manner and making the space with mosquito, plastic subcutaneous tunnellers for insertion of the central lines.

In our case we used trocar intercostal drain for the purpose of creation of the subcutaneous space because of the following reasons:

1. Its similarity with the most commonly used subcutaneous stainless steel tunnellers.

2. Its ease of availability in all the setups.

3. Its cost.

4. It's easy maneurability.

5. Its availability as ready to use sterile pack.
We were able to pass the trocar intercostal drain in the subcutaneous plane and guide the lower end of the shunt. The time required for creation of the space and guiding the lower end was 30 seconds which was the standard time required in creation of the space. All the cases could be easily performed using this method. The results were satisfactory,

\section{Conclusion}

We conclude that trocarintercostal drain is an easily available alternative for creation and insertion of subcutaneous part of the ventriculoperitoneal shunt. Its use can be popularized for insertion of ventriculoperitoneal shunts even in setups with limited facilities because it is easily available and cost effective.

\section{References}

1. Welch K. The intracranial pressure in infants. J Neurosurg 1980;52:693-9.

2. Ahmed M, Asif M, Ahmed N, Raja IA. The role of Surgery in the management of hydrocephalus. Ann King Edward Med Coll 1998;4:50-2. 